Centro, periferia e territorio fra amministrazione e frontiere: alcune considerazioni sugli Stati sabaudi e l'Arco Alpino Occidentale

Centre, périphérie et territoire entre administration et frontières: quelques réflexions sur les États de Savoie et l'arc alpin occidental

Center, Periphery and Territory between Administration and Boundaries. Observations on Sabaudian States and Western Alps

Michele Rosboch

OpenEdition

Journals

Edizione digitale

URL: https://journals.openedition.org/cei/10356

ISSN: 2260-779X

Editore

UGA Éditions/Université Grenoble Alpes

Edizione cartacea

ISBN: 978-2-37747-342-7

ISSN: 1770-9571

Notizia bibliografica digitale

Michele Rosboch, «Centro, periferia e territorio fra amministrazione e frontiere: alcune considerazioni sugli Stati sabaudi e l'Arco Alpino Occidentale», Cahiers d'études italiennes [Online], 34 | 2022, online dal 03 mars 2022, consultato il 27 janvier 2023. URL: http://journals.openedition.org/cei/10356

Questo documento è stato generato automaticamente il 27 janvier 2023.

All rights reserved 


\title{
Centro, periferia e territorio fra amministrazione e frontiere: alcune considerazioni sugli Stati sabaudi e l'Arco Alpino Occidentale
}

\author{
Centre, périphérie et territoire entre administration et frontières : quelques \\ réflexions sur les États de Savoie et l'arc alpin occidental \\ Center, Periphery and Territory between Administration and Boundaries. \\ Observations on Sabaudian States and Western Alps
}

Michele Rosboch

\section{Cenni introduttivi}

1 Da sempre le vicende dei rapporti centro/periferia e il regime giuridico delle comunità locali hanno rappresentato un nodo significativo degli assetti giuridici degli Stati, particolarmente in epoca moderna, nonché nella situazione attuale, richiamando l'attenzione dei giuristi, ma non soltanto.

2 A queste vicende sono legate, infatti, sia questioni generali (attinenti, per esempio alla forma stessa della compagine statale o delle relazioni interstatuali), sia aspetti più specifici, a partire dalla considerazione da parte del governo centrale dei cosiddetti 'corpi intermedi' o - con riguardo al contesto degli Stati sabaudi - le forme di sviluppo delle relazioni transfrontaliere ${ }^{1}$.

Inoltre, i rapporti centro/periferia costituiscono un importante banco di prova delle diverse concezioni giuridico-politiche e della capacità degli ordinamenti - statuali e non - di regolare situazioni complesse, che toccano profondamente la vita quotidiana dei singoli e delle comunità, avendo riguardo pure a risalenti tradizioni e sentite identità locali e collettive, così come alle interazioni fra realtà politiche confinanti ${ }^{2}$. 
4 Tutto ciò è rinvenibile anche negli sviluppi storici degli Stati sabaudi (nella loro costitutiva dimensione transfrontaliera) e particolarmente nel passaggio fra l'Antico regime e la Restaurazione, in cui emerge come: "Gli Stati sabaudi fino al 1848 non furono mai un "État-nation", ma una comunità di territori, che divenivano Stati (e non Stato, al singolare) grazie alla comune sovranità, fonte riconosciuta del diritto e del comando» ${ }^{3}$.

5 In un tale assetto la dimensione transfrontaliera è rilevante e forse costitutiva dell'assetto e delle vicende degli Stati sabaudi e delle intense relazioni che proseguono anche dopo la cessione di Nizza e Savoia alla Francia e la successiva unificazione italiana ${ }^{4}$.

6 Le vicende legate all'Arco alpino occidentale e quelle dei rapporti centro-periferia assumono inoltre un volto di particolare interesse in correlazione al tema dei confini e delle frontiere; riprendendo, infatti, la suggestiva lezione di Giorgio Lombardi i rapporti fra i poteri si collegano anche alla presenza o meno di 'frontiere' cetuali o comunitarie, che per molti secoli hanno caratterizzato la situazione istituzionale anche al di là dei confini fisici e geografici ${ }^{5}$.

7 Tutto ciò assume particolare rilievo nel microcosmo degli stati sabaudi e, soprattutto, per l'area cuneese fortemente caratterizzata dalla presenza di comunità montane e di rapporti transfrontalieri assai importanti, alla quale lo stesso Giorgio Lombardi ha dedicato alcuni studi di particolare rilievo ${ }^{6}$.

\section{Un percorso storico-istituzionale}

8 In senso storico, è indubbio che il volto degli ordinamenti locali della penisola in generale, e degli Stati sabaudi in particolare, nell'Antico Regime risentono ancora, per molti versi, del diffuso pluralismo istituzionale medievale, in cui il prevalente valore degli assetti comunitari costituiva l'ossatura dell'intera società, secondo una visione per cui anche le relazioni transfrontaliere assumono una veste di rilievo ${ }^{7}$.

9 Numerose tracce profonde di tale quadro permangono poi anche in epoca moderna, misurandosi con il protagonismo dei nascenti Stati, nei quali in molti episodi si misura una crescente conflittualità fra i sovrani e le autonomie locali e fra nuove tendenze centralistiche e antichi privilegi territoriali ${ }^{8}$.

10 Facendo un affondo sul Piemonte e sull'area cuneese in particolare emerge, a partire dal tardo medioevo e nella prima epoca moderna, l'idea di una vera e propria «rete giuridica», caratterizzata da una sorta di intersezione fra giurisdizioni particolari di diverso peso e rilievo sia di derivazione comunale, sia d'indole feudale'.

11 In tale 'groviglio' si affermano alcune realtà egemoni a livello locale (come Alba e Asti), capaci di attrarre a sé zone significative del proprio districtus, relazionandosi anche con le realtà signorili operanti: su tutte il declinante marchesato di Saluzzo e l'emergente ducato di Savoia ${ }^{10}$.

12 Di particolare rilievo sono anche le vicende che portano ad accordi e patti di dedizione da parte di alcune comunità montane operanti in ambiti transfrontalieri (come quelle della Val Maira, nel cuneese), che ottengono in questo modo il riconoscimento di alcune franchigie e di una certa autonomia economica ${ }^{11}$. 
13 Soprattutto a partire dal XVI secolo cresce l'influsso della dominazione sabauda, con una tendenza all'accentramento delle funzioni e alle riduzioni del peso delle autonomie, pur in una logica ancora plurale e per certi versi caratterizzata da prospettive di tipo cetuale e feudale, con la prevalenza dei legami personali ${ }^{12}$.

Un aspetto assai significativo di questo permanente «policentrismo» è l'assenza di una città dominante nei domini sabaudi, ove permangono per secoli vere e proprie «capitali periferiche», capaci di catalizzare gli interessi dei propri territori e attrarre localmente numerose attività economiche ${ }^{13}$.

Risulta poi il crescere delle prerogative attribuite al Senato di Piemonte nell'effettuare una sorta di controllo di conformità delle leggi locali allo ius generale principesco e la successiva sottrazione alle magistrature cittadine della prerogativa di esercitare il giudizio di sindacato sulle magistrature comunali.

ltimo - nel contesto delle riforme degli ordinamenti processuali operata da Emanuele Filiberto con il libro Terzo e il libro Quarto degli Ordini Nuovi - viene stabilita d'autorità la nullità delle norme cittadine in materia criminale: ormai la materia penale è attratta completamente nella legge comune e sottratta all'autonomia locale ${ }^{14}$.

17 In linea generale, ancora Giorgio Lombardi ha ulteriormente illustrato tali dinamiche attraverso l'analisi delle vicende della Guerra del sale e delle autonomie comunali dell'area cuneese in epoca moderna; ne emerge un quadro estremamente complesso, ricco di relazioni interistituzionali e all'insegna di un ancora diffuso pluralismo organicista, in cui - peraltro - si vedono all'opera spinte accentratrici e nuove forme di controllo dei territori messe in atto dai sovrani ${ }^{15}$.

Si possono pertanto rilevare presenze di numerose frontiere fra ordinamenti, fra cui quelli cetuali, più o meno aperte o 'porose', con particolare rilievo per l'assetto giuridico delle comunità montane e le relazioni transfrontaliere ${ }^{16}$.

19 Proprio il livello locale, in specie comunale, costituisce l'entità di riferimento per la gran parte delle attività quotidiane, e la stessa giustizia si amministra per lo più in questo spazio comunitario, a sua volta inserito in contesti aggregati maggiori, fino a giungere - per successive e necessarie mediazioni - alla dimensione statale in cui opera e da cui si diparte l'esercizio della sovranità regia ${ }^{17}$.

20 Con specifica attenzione al percorso istituzionale degli Stati sabaudi, assume importanza notevole il Regolamento dei pubblici di Vittorio Emanuele III del 1775, volto a operare una prima (ma epocale) razionalizzazione del regime giuridico dei comuni del Regno; pur prevedendo numerose eccezioni per le città maggiori (fra cui Torino, Alessandria, Novara, Nizza e Chambéry) il provvedimento è un momento di non ritorno verso l'accentramento e il ridimensionamento degli spazi di autonomia, che interessa anche le aggregazioni e le istituzioni collocate nello spazio dell'arco alpino occidentale ${ }^{18}$.

21 Si tratta di una normativa assai complessa, volta soprattutto a operare un controllo delle spese dei comuni - il che sarà una costante anche dei progetti e degli interventi successivi - e limitare, per quanto possibile, l'ingerenza sulle comunità locali da parte di nobili e feudatari ${ }^{19}$.

22 In questo senso, se le frontiere interne dello Stato tendono a cadere anche attraverso questi interventi regi, restano ancora saldi, seppur erosi, i confini cetuali, anche 
all'interno delle stesse realtà locali, così come gli ambiti propri di giurisdizioni extrastatuali, come quella ecclesiastica ${ }^{20}$.

Tra l'altro la linea che porterà alla caduta delle frontiere 'interne' negli Stati comporta il rafforzamento di quelle esterne, con la ricerca di appigli naturali e geografici a presidio della stessa sovranità: in tal senso assume un certo rilievo l'area alpina, che in alcuni tratti è completamente nell'area degli Stati sabaudi, ma in altre zone viene a costituire il naturale presidio di confine. In particolare si afferma il ruolo duplice delle zone alpine, da certi punti di vista presidio e confine, da altri via di comunicazione e di condivisione di attività economiche comuni e conseguenti e prassi e consuetudini condivise ${ }^{21}$.

Più in generale, la dialettica fra centro e periferia, la decisa spinta verso l'accentramento delle funzioni e delle decisioni politiche e l'innalzamento di barriere (anche fisiche, spesso in area montana) a presidio dei confini costituiscono - pur con sfumature diverse e qualche eccezione - una cifra essenziale nell'evoluzione dello Stato moderno.

Come ha ben osservato Lorenzo Ornaghi: «Ora, che l'intera vicenda dello Stato moderno possa essere fatta coincidere con il faticoso tentativo di costruire (e poi di salvaguardare) un unico centro della titolarità e della gestione del potere politico, è interpretazione che la stragrande maggioranza della storiografia non sembra disposta a revocare in dubbio» ${ }^{22}$.

Proseguendo nel percorso storico, il passaggio napoleonico incide in profondità anche sugli assetti delle istituzioni locali e periferiche nei territori degli Stati sabaudi, realizzando l'inserimento dei territori sabaudi nella nuova organizzazione francese, fortemente accentrata, e impostata sulla preponderanza delle prefetture sulle istituzioni locali ${ }^{23}$.

Inoltre, è proprio in questo periodo storico che si perfeziona - anche se non in modo irreversibile per i territori sabaudi - la sostanziale liquidazione delle comunità politiche, delle corporazioni e dei corpi intermedi, fra i quali si annoverano anche numerose aggregazioni collocate nell'area alpina ${ }^{24}$.

Nello specifico, com'è ovvio, l'appartenenza del Piemonte alla Francia (con le sue articolazioni amministrative periferiche) omologa anche le amministrazioni montane e dell'Arco alpino occidentale ${ }^{25}$.

29 Con il ritorno dei Savoia al potere si procede rapidamente al ripristino della normativa e degli assetti amministrativi precedenti al 1799, pur con qualche modifica e innovazione; in special modo a partire dai primi anni del regno di Carlo Felice si mettono in cantiere alcune ipotesi di riforma delle amministrazioni comunali anche in seguito a un'accurata indagine sulle necessità delle comunità locali avviata dagli intendenti sabaudi ${ }^{26}$.

30 Gli interventi di Carlo Felice prima (a partire dal significativo ritorno ai giuramenti dei nobili e degli ecclesiastici imposti nel biennio 1821-1822) ${ }^{27}$ e di Carlo Alberto poi (a iniziare dall'istituzione del Consiglio di Stato nel 1833$)^{28}$ vanno nell'ottica di assicurare una sorta di controllo centrale operato attraverso l'amministrazione delle comunità - specialmente per quanto concerne le spese - e avvalendosi in primo luogo delle capacità del ceto nobiliare dirigente, soprattutto locale ${ }^{29}$.

31 Pur in un contesto caratterizzato da un certo accentramento, non si riscontra di per sé una ostilità ideologica nei confronti delle autonomie locali, soprattutto quelle più 
risalenti, considerate anche come una possibile limitazione delle ben più radicali idee 'liberali' ${ }^{30}$. 
41 Più significativo per le innovazioni che contiene è il Regio editto 27/11/1847, ispirato dall'avvocato novarese Giacomo Giovanetti, che ridisegna le amministrazioni comunali con notevoli aperture, tanto da essere considerata una sorta di anticipazione della stessa futura carta costituzionale albertina, che, in verità, ne sancirà il superamento ${ }^{40}$. È con lo Statuto carloalbertino e la successiva legge sulle amministrazioni locali del 1848 che si realizza il superamento della concezione 'eclettica' e ancora organicistica della Restaurazione, che vedeva nelle comunità territoriali l'ambito, seppur limitato rispetto ai fasti del passato, di garanzia dei diritti e della rappresentatività locale ${ }^{41}$.

43 Il passaggio al costituzionalismo implica l'assunzione da parte della costituzione (e della stessa monarchia parlamentare) del compito di tutelare i cittadini e da parte del parlamento di assicurare la rappresentanza dei territori e dei diversi interessi, riducendo le prerogative degli enti locali alle funzioni amministrative decentrate, prevalenti rispetto a quelle di governo delle comunità, pur salvaguardando alcuni - minimi - diritti democratici ${ }^{42}$.

44 Assai scarso è lo spazio per le istituzioni locali intermedie di stampo territoriale più ampio ad esempio regionale, con conseguente impostazione di tipo federalista, occupandosi piuttosto della riorganizzazione dei comuni e di forme di aggregazione sovracomunale ${ }^{43}$.

45 La riforma Rattazzi del 1859, esito di una complessa gestazione e di un significativo dibattito politico e parlamentare, afferma la prevalenza del centro e il forte controllo governativo sulle istituzioni locali, riconoscendo scarse libertà a livello locale, con autonomia amministrativa (ma non politica!) e senza spazio per altri enti intermedi autonomi, vanificando progetti più liberali: dal 1848 al 1859 si consuma quindi la liquidazione di tutte le frontiere interne al Regno di Sardegna, avviandosi verso il modello liberale, individualista ed egualitario, in cui scarsa attenzione troveranno le aree alpine con le loro tradizioni e 'specialità't4.

\section{Problematiche aperte fra Stato, territori e comunità}

Le pur sommarie considerazioni proposte hanno evidenziato l'interesse del microcosmo sabaudo, proprio per la sua multiformità, in cui assumono particolare rilievo anche le aree dell'Arco alpino occidentale, quale spazio di comunicazione e di frontiera, oltre che di elaborazione giuridica e sociale.

Peraltro, al Regno di Sardegna ottocentesco quale diretto 'erede' degli Stati sabaudi di Antico Regime si deve in gran parte anche l'opera di unificazione nazionale, offrendo spunti storiografici di eccezionale rilievo e interesse anche per comprendere alcuni nodi dell'epoca contemporanea, come è stato autorevolmente sottolineato ${ }^{45}$.

In questo senso, il recente centocinquantenario dell'unificazione giuridica e amministrativa del Regno d'Italia (1865-2015) ha consentito di richiamare alcune riflessioni generali circa il percorso compiuto dal periodo preunitario alle scelte politiche e giuridiche compiute dopo la proclamazione dell'Unità, che scontano per molti versi la loro derivazione dalla più risalente tradizione sabauda preunitaria ${ }^{46}$.

49 Al contempo, la stessa ricorrenza ha rappresentato lo spunto per riprendere, anche alla luce di complesse problematiche attuali, il tema della dialettica centro/periferia e l'efficacia delle modalità di superamento del centralismo, attuate in Italia negli ultimi 
decenni attraverso forme di federalismo 'differenziato' di non facile applicazione e di non immediata accettazione da parte dell'ordinamento e delle stesse istituzioni ${ }^{47}$; come ha osservato Gian Savino Pene Vidari: «Dopo quasi un secolo di centralizzazione, conclusasi in modo oppressivo ed autoritario, ci si è atteso molto da un mutamento notevole di prospettiva, con 'molto meno Stato', più autonomia locale (impersonata nell'avvio generale delle Regioni), mentre veniva emergendo un terzo più generale livello di integrazione comunitaria europea» ${ }^{48}$.

50 È innegabile, peraltro, che anche alcune attuali problematiche non siano frutto soltanto del difficile contesto istituzionale e politico odierno, ma scontino scelte più risalenti, le cui tappe (a partire dall'Antico Regime) sono state oggetto di accurate indagini e articolate - seppur diverse - valutazioni storiografiche ${ }^{49}$.

51 Tale sviluppo si presenta poi, dopo l'Unità, assai complesso, fino al recupero - soprattutto negli ultimi decenni del secolo XIX - di una più diffusa socialità del fenomeno giuridico; il secolo XX culmina poi nella nuova valorizzazione delle realtà locali, a partire dall'attuazione del regionalismo previsto dalla Carta costituzionale dopo ampie e articolate discussioni, sviluppatosi all'interno di un mondo globalizzato e con la crescente 'crisi' dello Stato (nazionale) ${ }^{50}$.

52 A ben vedere, alcuni dei nodi della successiva evoluzione post-statutaria e unitaria dei rapporti centro-periferia erano già presenti (almeno in nuce) nei progetti e nelle sottostanti riflessioni nel periodo della Restaurazione e nel passaggio all'ordinamento costituzionale, sempre a cavallo fra uniformità e difesa dei fenomeni locali e comunitario-cetuali, incluse le realtà assai particolari e ricche di tradizioni quali sono le aree alpine ${ }^{51}$.

Invece, in senso generale e in un'ottica di lungo periodo si possono vedere le evoluzioni dei rapporti fra centro e periferia, così come quelle dell'efficacia dei meccanismi di rappresentanza $^{52}$ : sembra emergere a livello generale una sorta di crisi della globalizzazione, che si affianca alla più risalente messa in discussione dello Stato come unico interprete legittimo della vita sociale, senza dimenticare l'odierna e preoccupante difficoltà di reclutamento e legittimazione delle classi dirigenti e di governo ${ }^{53}$.

Al contempo, si stanno affermando - al di là di formule istituzionali semplificate e promesse di ritorni a forme di democrazia diretta - visioni di tipo localistico e di recupero delle identità provenienti dalle tradizioni e dalle diverse componenti sociali, peraltro non prive di qualche ambiguità ${ }^{54}$ : il latente conflitto dell'epoca moderna e contemporanea fra Stato e società, così come la tensione fra centro e periferia, si riflette anche nella vita politica, rendendo difficile individuare i criteri condivisi della convivenza e il significato di bene comune $\mathrm{e}^{55}$.

Le regole, i valori e le stesse politiche sono chiamate a concorrere - il più possibile per assicurare la sostenibilità delle istituzioni nello spazio pubblico, come ben ha evidenziato Lorenzo Ornaghi con riferimento alla «[...] molteplicità di poteri, autorità, istituzioni, che oggi compone e regola ogni organizzazione della convivenza politica, ai livelli più differenti della sua articolazione e lungo lo smobilitarsi progressivo delle demarcazioni più tradizionali tra ciò che è "dentro" e ciò che è "fuori", oltre che all'interno stesso, dell'unità di una sintesi politico-statale» ${ }^{56}$.

In questa direzione, concludendo con una riflessione sull'attualità, anche nel contesto della pandemia, mi permetto di avanzare come tema quello del valore dei soggetti 
'intermedi', oggi particolarmente attivi nel cosiddetto privato sociale e attenti al recupero dei valori tradizionali così come dei legami sociali ${ }^{57}$.

Proprio questi possono forse rappresentare un punto di equilibrio (seppur dinamico) fra centro e periferia, così come un elemento di stabilizzazione sociale e di recupero del valore della partecipazione civica e politica ${ }^{58}$.

\section{NOTE}

1. Le presenti considerazioni, che traggono spunto dai lavori del convegno «Pridaes» svoltosi a Torino il 28-29 ottobre 2021 sul tema «La legge, espressione della sovranità, di fronte alle autonomie negli antichi Stati Sabaudi», riprendono anche alcune osservazioni contenute in M. Rosboch, «Con somma cautela e maturità di giudizio». Centro, periferia e comunità in un progetto di riforma nella Restaurazione sabauda, Napoli / Torino, ESI, 2019.

2. Per tutte queste considerazioni, vd. P. Aimo, Il centro e la circonferenza: profili di storia delle amministrazioni locali, Milano, Franco Angeli, 2005.

3. P. Bianchi e A. Merlotti, Storia degli Stati sabaudi (1416-1848), Brescia, Morcelliana, 2017, p. 47; cfr. anche G. Tabacco, Lo Stato sabaudo nel Sacro Romano Impero, Torino, Paravia, 1939.

4. Cfr. fra i molti F. Peirone et al., Per Torino da Nizza e Savoia. Le opzioni del 1860 per la cittadinanza torinese da un Fondo dell'Archivio Storico della Città di Torino, a cura di G. S. Pene Vidari e R. Roccia, Torino, Centro Studi Piemontesi, 2011.

5. G. Lombardi, Spazio e frontiera tra eguaglianza e privilegio: problemi costituzionali fra storia e diritto, in Id., Scritti scelti, a cura di E. Palici di Suni e S. Sicardi, Napoli, ESI, 2012, pp. 539-559 (già in Scritti su le fonti normative e altri temi di vario diritto in onore di Vezio Crisafulli, vol. II, Padova, Ceda, 1986, pp. 477-495; alcune osservazioni, qui riprese, in M. Rosboch, Frontiere e confini «porosi» nel tempo $e$ nello spazio: centro, periferia e autonomia regionali alla luce del pensiero di Giorgio Lombardi, in «Politica.eu», Pagine nuove (2021), <www.rivistapolitica.eu/frontiere-e-confino-porosi-neltempo-e-nello-spazio-centro-periferia-e-autonomie-regionali-alla-luce-del-pensiero-di-giorgiolombardi/>.

6. G. Lombardi, I Comuni della provincia di Cuneo nello Stato Sabaudo: problemi evolutivi delle autonomie locali, in Id., Scritti scelti, cit., pp. 139-165; sull'opera del giurista torinese, significativo Giorgio Mario Lombardi. Giurista, storico e amministratore. A dieci anni dalla scomparsa, a cura di S. Sicardi, G. Mola di Nomaglio e M. Fessia, Torino, Centro Studi Piemontesi, 2021.

7. Di un certo interesse è l'area saluzzese, in cui si avvicendano, dal sec. XV, il Marchesato e i Savoia, mantenendo in vita significativi scambi nell'area alpina: cfr. fra i molti: A. A. Mola (a cura di), Il Marchesato di Saluzzo da stato di confine, a confine di stato, a Europa, Atti del convegno per il 4. centenario del Trattato di Lione (Saluzzo, 30 novembre-1 dicembre 2001), Foggia, Bastogi, 2003; in generale, G. Lombardi (a cura di), Partecipazione e autonomia nelle territorialità dell'area alpina occidentale. Profili storici e giuridici, Milano, Franco Angeli, 1988.

8. Per tutti, cfr. P. Bianchi e A. Merlotti, Storia degli Stati sabaudi, cit., in specie pp. 63-102; cfr. anche B. A. Raviola (a cura di), Lo spazio sabaudo. Intersezioni frontiere e confini in età moderna, Milano, Franco Angeli, 2007.

9. Com'è noto, è il concetto di «iurisdictio» a descrivere tale contesto intimamente plurale; per tutti, P. Costa, Iurisdictio. Semantica del potere politico nella pubblicistica medievale (1100-1433), Milano, Giuffrè, 2002 (1969); L. Mannori e B. Sordi, Storia del diritto amministrativo, Bari / Roma, Laterza, 
2001, pp. 5-221. La situazione sabauda è caratterizzata dalla presenza della consolidazione di Amedeo VIII risalente al 1430, di cui si è data di recente - per impulso di Gian Savino Pene Vidari - una pregevole edizione critica: cfr. La loi du Prince. La raccolta normativa sabauda di Amedeo VIII (1430), a cura di M. Caesar e F. Morenzoni, Torino, Deputazione subalpina di storia patria, vol. I-II, 2019.

10. Efficace quadro in G. Lombardi, I Comuni della provincia di Cuneo, cit., pp. 145-153; in generale, A. Barbero, Il Ducato di Savoia: amministrazione e corte di uno Stato franco-italiano (1416-1536), Roma / Bari, Laterza, 2002.

11. Cfr. E. Coscia, Aspetti delle autonomie locali nella provincia di Cuneo in prospettiva storica, in S. Sicardi (a cura di), Le autonomie territoriali e funzionali nella provincia di Cuneo in prospettiva transfrontaliera (alla luce del principio di sussidiarietà), Napoli, ESI, 2011, pp. 63-95.

12. Considerazioni di rilievo in P. Merlin, C. Rosso e G. Symcox, Il Piemonte sabaudo. Stato e territori in età moderna, Torino, Utet, 1994.

13. La centralità dei luoghi periferici e le capitali secondarie. Aspetti del potere locale nell'equilibrio europeo, colloquio franco-italiano, Cuneo, 28-29 settembre 1988.

14. Decreto 29.12.1559; sulle riforme in ambito processuale di Emanuele Filiberto, cfr. Il libro terzo degli 'Ordini Nuovi' di Emanuele Filiberto, nota e introduzione a cura di C.Pecorella, Torino, Giappichelli, 1988 e Il libro quarto degli 'Ordini Nuovi' di Emanuele Filiberto, a cura di C. Pecorella, Torino, Giappichelli, 1994; si osservi inoltre, che nel 1570 vengono riformati ancora in ottica 'localistica' gli statuti del comune di Mondovì (cfr. E. Coscia, Aspetti delle autonomie, cit., pp. 90-91). 15. Cfr. G. Lombardi, La Guerra del Sale: Caleidoscopio di una Historia, in Id. (a cura di), La Guerra del Sale (1680-1699). Rivolte e frontiere del Piemonte barocco, vol. I, Milano, 1988, pp.39-178. Con riferimento sempre agli Stati sabaudi, ricade in questo ambito anche l'istituto della «interinazione» degli atti sovrani: G. Lombardi, Note sul controllo degli atti del sovrano negli Stati Sabaudi ad opera delle supreme magistrature nel periodo dell'assolutismo, in Id., Scritti scelti, cit., pp. 73-114.

16. Per interessanti notazioni interdisciplinari sul tema, si vedano: F. Ferlaino, D. Iacobucci e C. Tesauro (a cura di), Quali confini? Territori tra identità e integrazione internazionale, Milano, Franco Angeli, 2017; in prospettiva storica, significativi: A. M. Hespanha, L'espace politique dans l'ancien régime, «Boletin da Facultatde de Direito-Universidade de Coimbra», vol.58, $\mathrm{n}^{\circ}$ 2, 1982, pp. 455-510 e P. Marchetti, De iure finium: diritto e confini tra tardo medioevo ed età moderna, Milano, Giuffrè, 2001.

17. In tale contesto s'inserisce la riflessione sull'importanza dei corpi intermedi e della loro evoluzione storico-giuridica, su cui cfr. per tutti P. Grossi, Le comunità intermedie tra moderno e posmoderno, a cura di M. Rosboch, Genova, Marietti, 2015.

18. Nello stesso periodo storico si registrano significativi interventi sovrani nei confronti delle autonomie locali anche in Toscana: cfr. L. Mannori, Dopo la riforma. Comunità locali e rappresentanza in Toscana al tramonto dei Lumi, "Quaderni fiorentini per la storia del pensiero politico moderno", vol. 48, 2019, pp. 221-253.

19. G. Lombardi, Le origini, in M. Dogliani, J. Luther e A. Poggi (a cura di), Lineamenti di diritto costituzionale della Regione Piemonte, Torino, Giappichelli, 2018, pp. 4-8.

20. Sempre Giorgio Lombardi ha osservato con molta acutezza, a proposito della libertà religiosa, che essa assume in Antico Regime soprattutto il volto di una difesa delle prerogative (e delle frontiere...) delle comunità, mentre a partire dal secolo XIX l'attenzione si sposta sulla tutela dei diritti individuali: si veda G. Lombardi, La libertà religiosa, in Id., Scritti scelti, cit., pp. 435-469); molto significativa è anche la ricostruzione di Arturo Carlo Jemolo a cui Giorgio Lombardi fa spesso riferimento nei suoi scritti: A. C. Jemolo, Chiesa e Stato in Italia negli ultimi cento anni, Torino, Einaudi, 1948.

21. Ha costituito e costituisce un significativo ambito di elaborazione scientifica e culturale su questi temi il «Centro di Studi sull'Arco Alpino Occidentale», fondato negli anni Ottanta del 
secolo scorso da studiosi italiani e francesi con importanti attività convegnistiche ed editoriali, di cui si può leggere una sintesi in D. Grange, Regard sur trente ans de coopération entre les Universités de Grenoble et de Turin. Les colloques franco-italiens d'études alpines, in A.Crosetti e M. Rosboch (a cura di), Le dinamiche del cambiamento. Cultura, cittadinanza nelle regioni alpine occidentali tra età moderna e globalizzazione, Torino, Stampatori, 2009, pp. 9-24.

22. L. Ornaghi, "Crisi» del centro statale e "disseminazione" di centri politici. Note su un indice di trasformazione dello Stato moderno, "Quaderni sardi di storia», vol. 4, 1983-1984, p. 49; cfr. anche, per significative osservazioni generali, P. Cappellini, Critica dello Stato moderno e consistenza della politica. Per Lorenzo Ornaghi: un percorso di lettura, in P. Colombo, D. Palano e V. E. Parsi (a cura di), La forma dell'interesse. Studi in onore di Lorenzo Ornaghi in occasione del suo settantesimo compleanno, Milano, Vita e Pensiero, 2018, pp. 117-140.

23. Sull'evoluzione delle vicende delle amministrazioni locali, in generale, A. Petracchi, Le origini dell'ordinamento comunale e provinciale italiano. Storia della legislazione piemontese sugli enti locali dalla fine dell'antico regime al chiudersi dell'età cavouriana (1770-1861), Vicenza, Neri Pozza, 1962; più nello specifico, fra i molti: S. Mannoni, L'unificazione italiana e l'accentramento napoleonico: miti e realtà, «Le Carte e la Storia», a. III, fasc. 2, 2000, pp.30-37 e C. Ghisalberti, L'influsso della Francia napoleonica sul sistema giuridico e amministrativo dell'Italia, in Id., Modelli costituzionali e stato risorgimentale, Roma, Carucci, 1987, pp. 37-54.

24. Per tutti, A. Pennini, Dalla Societas al Citoyen. Lo anéantissement delle comunità intermedie nel percorso costituzionale della Francia rivoluzionaria, in M. Rosboch (a cura di), Le comunità intermedie e l'avventura costituzionale. Un percorso storico-istituzionale, Torino, Heritage, 2017, pp. 71-98; sull'area alpina particolarmente rilevante è la vicenda degli usi civici, su cui cfr. per tutti A. Crosetti, Gli "usi civici» tra passato e presente in una dimensione europea, in A. Crosetti e M. Rosboch (a cura di), Le dinamiche del cambiamento, cit., pp. 25-36.

25. Spunti interessanti sull'area delle Alpi marittime in O. Vernier (a cura di), Du Comté du Nice aux Alpes-Maritimes. Les représentations d'un espace politique et culturel dans l'histoire, Nizza, Serre, 2000.

26. Mi permetto di rinviare a M. Rosboch, Fra angustie di coscienza e ordine politico, vol. I: Il giuramento degli ecclesiastici all'inizio del regno di Carlo Felice (1821-1822), Milano, Ledizioni, 2017.

27. Su cui cfr. M. Rosboch, «Con somma cautela e maturità di giudizio», cit., passim.

28. Si vedano: Atti del convegno celebrativo del $150^{\circ}$ anniversario della istituzione del Consiglio di Stato, Milano, Giuffrè, 1983 e C. Franchini (a cura di), Il Consiglio di Stato nella storia d'Italia, Torino, Utet, 2011.

29. Cfr. E. Genta, Dalla Restaurazione al Risorgimento. Diritto, diplomazia, personaggi, Torino, Giappichelli, 2012, in specie pp. 14-25.

30. E. Genta, Una rivoluzione liberale mancata. Il progetto Cavour-Santarosa sull'amministrazione comunale e provinciale (1855), Torino, Deputazione subalpina di storia patria, 2000, pp. 119-144.

31. Si veda per tutti A. Petracchi, Le origini dell'ordinamento, cit., pp. 43-58.

32. Lo ha evidenziato G.S. Pene Vidari, Aspetti del regolamento forestale albertino, in P.Caroli, P. Corti e C. Pischedda, L'agricoltura nel Piemonte dell'800. Atti del seminario in memoria di Alfonso Bogge (Torino, 2 dicembre 1989), Torino, Centro Studi Piemontesi, 1991, pp.35-71; Id., La normativa forestale da Carlo Felice a Carlo Alberto, in Per un museo dell'agricoltura in Piemonte, vol. V: Il bosco e il legno, Torino, Associazione Museo dell'Agricoltura del Piemonte, 1987, pp. 211-227 e, più in generale, Id., Autodisciplina e normazione nella storia dell'ambiente, in M. Ortolani (a cura di), Protection et valorisation des ressources naturelles dans les États de Savoie, Atti del convegno internazionale di Cuneo (6-7 ottobre 2011), Nizza, Serre, 2014, pp. 3-13.

33. Sui principali aspetti politico-giuridici della Restaurazione, per tutti: G. S. Pene Vidari, Studi e prospettive recenti di storia giuridica sul Piemonte della Restaurazione, «Studi Piemontesi», vol. XII, fasc. 2, 1983, pp. 416-422; M. Meriggi, Gli stati italiani prima dell'Unità. Una storia istituzionale, Bologna, Il Mulino, 2002; per un quadro recente, mi permetto di richiamare M. Rosboch, Profili 
della recente storiografia giuridica sul primo Ottocento, in B. A. Raviola, C. Rosso e F. Varallo (a cura di), Gli spazi sabaudi. Percorsi e prospettive della storiografia, Roma, Carocci, 2018, pp. 259-270.

34. Cfr. G. Lombardi, Il Consiglio di Stato nel quadro istituzionale della Restaurazione, in Id., Scritti scelti, cit., pp. 115-138.

35. Cfr. M. Rosboch, «Con somma cautela e maturità di giudizio», cit., pp. 27-80.

36. La frase è contenuta in: «Istituzioni e stabilimenti pubblici tendenti a procurare il maggior grado di prosperità dei Regi Stati. Transunto analitico delle relazioni degl'Intendenti state trasmesse a tale riguardo alla regia segreteria di segreteria di Stato» (in ASTo, Corte, Paesi in genere per Province, mazzo 7, fascicolo 18, p. 31); cfr. M. Rosboch, «Con somma cautela e maturità di giudizio", cit., pp. 48-50.

37. «Istruzione per l'amministrazione dei Comuni approvata da Sua Maestà. In data $1^{\circ}$ d'aprile 1838» (in «Raccolta degli atti del Governo di Sua Maestà il Re di Sardegna», ${ }^{\circ}$ 6, 1838, pp. 69-263), riprodotta da A. Petracchi, Le origini dell'ordinamento, cit., vol. II, pp. 158-248, su cui cfr. E. Genta, L'«Istruzione Pralormo» del 1838, in M. Ortolani, O. Vernier e K. Deharbe (a cura di), Intendants et Intendance en Europe et dans les États de Savoie. XVII ${ }^{e}-\mathrm{XVIII}{ }^{e}$ siècles, Nizza, Serre, 2015, pp. 449-457.

38. Non va dimenticato che le disposizioni comunali degli antichi statuti cessano di avere valore con l'entrata in vigore del codice civile albertino del 1837.

39. «Uno dunque è il rapporto di rappresentatività istituzionale, ma la stessa tendenza, chiaramente organicistica non si limita al profilo dei collegamenti con i corpi locali (è chiara in questo una derivazione delle ideologie tipiche della restaurazione caratterizzate da un istituzionalismo che si tinge di colori corporativi) ma comprende anche profili individualistici del rapporto, giungendo a fare del Consiglio il portavoce presso la Corona delle doglianze, dei torti, degli abusi sia dell'Amministrazione attiva, sia dei corpi sociali» (G. Lombardi, Il Consiglio di Stato, cit., p. 135).

40. Per tutti C. Pecorella, Giacomo Giovanetti e la riforma delle amministrazioni locali sabaude del 1847, in Id., Studi e ricerche di storia del diritto, Torino, Giappichelli, 1995, pp. 619-627.

41. In generale, per tutti, L. Mannori, Costituire l'Italia. Il dibattito sulla forma politica nell'Ottocento preunitario, Pisa, Pacini, 2019, pp. 138-192.

42. "Quel che preme rilevare è che la rivoluzione costituzionale del' 48 non segnò solo la fine virtuale dell'assolutismo, ma anche quella di un ordine politico tardo-medievale, compromesso ma non certo sradicato dalle riforme settecentesche e dall'esperienza napoleonica. L'essenza di tale ordine stava nella sua struttura intrinsecamente corporativa e composita; e parallelamente nel suo configurare lo Stato non come soggetto intenzionato, portatore di una volontà e di un programma totalizzanti, ma come il semplice contenitore di una pluralità di soggetti politici minori, di cui esso era chiamato a garantire l'equilibrio. La scommessa del biennio riformatore fu appunto quella di rimettere al passo questo "Stato di repubbliche" con i nuovi sviluppi del costituzionalismo occidentale senza sconfessarne i valori di fondo. Il che significava, in primo luogo, riuscire ad innestarvi sopra la dimensione della libertà politica (fino ad allora da esso completamente ignota), facendola scaturire da quelle libertà domestiche, periferiche e semiprivate, che da sempre ne avevano caratterizzato l'ordito» (L. Mannori, Costruire l'Italia, cit., p. 139).

43. Ancora Luca Mannori, in un'ottica assai vicina a quella di Giorgio Lombardi, osserva come: «L'antica figura dello Stato-consociazione venne così definitivamente archiviata; e con essa svanì pure l'alternativa federalista che, benché formalmente sepolta solo all'indomani dell'unità, costitù a tutti gli effetti la vera, grande vittima istituzionale della svolta quarantottesca» (L. Mannori, Costituire l'Italia, cit., p. 167).

44. Cfr. E. Genta, Una rivoluzione liberale mancata, cit., pp.167-205; cfr. anche in generale R. Romanelli, Centralismo e autonomie, in Id. (a cura di), Storia dello Stato italiano dall'Unità a oggi, Roma, Donzelli, 1995, pp. 126-186. 
45. «Il Piemonte è davvero un oggetto privilegiato d'osservazione non soltanto per la sua posizione a cavallo delle Alpi e per la sua consistenza plurinazionale, ma per il variare concreto nella dinamica storica di tutti quegli assi di questo rapporto centro/periferia, da quello più propriamente politico-amministrativo a quello linguistico, religioso, eccetera. Il riflettere quindi sul caso Piemonte ci deve impedire di cadere in un discorso astratto di modellistica, non importa se geografica o antropologica, per legarci alla storia concreta di uomini e di fenomeni collocati nel tempo» (P. Prodi, Introduzione, in C. Ossola, C. Raffestin e M. Ricciardi (a cura di), La frontiera da Stato a Nazione. Il caso Piemonte, Roma, Bulzoni, 1987, p. 13).

46. Per tutti, vd. G. S. Pene Vidari, Note e considerazioni su unità e unificazione italiana a 150 anni dall'unificazione legislativa e amministrativa, «Bollettino Storico-Bibliografico Subalpino», a. CXIII, fasc. II, 2015, pp. 519-566; cfr. anche F. Benvenuti e G. Miglio (a cura di), L'unificazione amministrativa e i suoi protagonisti. Atti del convegno celebrativo del centenario delle leggi amministrative di unificazione, 6 volumi, Vicenza, Neri Pozza, 1969 e $150^{\circ}$ dell'unificazione amministrativa italiana (legge 20 marzo 1865, n. 2248), "Storia Amministrazione Costituzione - Annale ISAP», vol. 23, 2015.

47. Fra i moltissimi: P. Aimo, Il centro e la circonferenza, cit, pp. 173-288; M. Nigro, Il governo locale, vol. I: Storia e problemi, Roma, Bulzoni, 1980; F. Bonini, Storia della pubblica amministrazione in Italia, Firenze, Le Monnier, 2004 e S. Cassese, Governare gli italiani. Storia dello Stato, Bologna, Il Mulino, 2014.

48. G. S. Pene Vidari, Note e considerazioni, cit., p. 566.

49. In proposito la rassegna è molto ampia; mi limito a richiamare, per tutti, E. Genta, Dalla Restaurazione al Risorgimento, cit., passim con bibliografia ivi richiamata.

50. In generale: O. Hintze, Stato e società, Bologna, Il Mulino, 1980; S. Rokkan, Stato, nazione e democrazia in Europa, Bologna, Il Mulino, 2002; P. Serra, La funzione dello Stato. Scienza giuridica europea e rapporti tra ordinamenti, Roma, Aracne, 2010; inoltre, P. Costa, Lo Stato immaginario. Metafore e paradigmi nella cultura giuridica fra Ottocento e Novecento, Milano, Giuffrè, 1986; da ultimo, molto importante: B.Sordi, Diritto pubblico e diritto privato. Una genealogia storica, Bologna, Il Mulino, 2020.

51. Guido Melis ha sintetizzato l'ambiguità complessiva anche del successivo centralismo italiano, che: «Si era invece subito configurato come un centralismo debole ("contrattato"), fondato su quello che Raffaele Romanelli ha definito felicemente come "il comando impossibile". Un modello nel quale il rapporto centro-periferia si era evoluto ambiguamente: centralistico l'ordinamento, basato sul primato assoluto, per legge, dello Stato sulle autonomie locali; ma al tempo stesso la prassi di un governo basata su uno scambio continuo quanto sotto traccia tra le istanze di integrazione dal basso e la politica assimilatrice del centro. Una filiera parallela di interconnessioni quasi impalpabili, perché espressa in un continuo do ut des, aveva sin dall'inizio supplito al deficit di egemonia del centralismo debole e ne aveva per così dire garantito la sopravvivenza» (G. Melis, Dalle piramidi alla rete: storia e trasformazioni delle istituzioni amministrative, «Le Carte e la Storia», a. XIX, fasc. 1, 2013, p. 5).

52. «[...] la contrapposizione antico-moderno conserva una vera nettezza di profilo solo sul piano culturale. Mentre quello dei fatti ci dice come l'essenza della statualità (che potremmo chiamare in blocco 'moderna' per distinguerla da quella medievale) consiste in questo tentativo plurisecolare di dirigere le collettività che popolano il proprio territorio essenzialmente tramite strumenti di verifica finanziaria. Tentativo caratterizzato da un arrestarsi di stasi, accelerazioni, regressi indotti da una dialettica centro-periferia assolutamente non unilineare né avviata su alcun percorso predefinito; ma tentativo altrettanto sicuramente capace di attribuire una cifra unitaria alla storia amministrativa, al di là non solo delle cesure epocali ma anche delle dimensioni e del rilievo dei singoli ordinamenti» (L. Mannori, Il sovrano tutore. Pluralismo istituzionale e accentramento amministrativo nel principato dei Medici (secc. XVI-XVIII), pp. 473-474); si veda anche L. Ornaghi, Sui confini dell'amministrazione e della politica odierna, "Amministrare», fasc. 2, 2016, pp. 299-309. 
53. Per tutti: A. Baldassarre, Globalizzazione contro democrazia, Roma / Bari, Laterza, 2002; C. Taylor, La democrazia e suoi dilemmi, Parma, Diabasis, 2014; A. Lijphart, Le democrazie contemporanee, Bologna, Il Mulino, 1984 e O. Höffe, La democrazia nell'era della globalizzazione, Bologna, Il Mulino, 2007 (1999); di grande utilità M. Fioravanti, Lezioni di diritto costituzionale. Le libertà fondamentali, le forme di governo, le costituzioni del Novecento, Torino, Giappichelli, 2021.

54. Cfr. B. Sordi, Oltre la dicotomia tra pubblico e privato: le origini del diritto sociale, in P. Colombo, D. Palano e V.E. Parsi (a cura di), La forma dell'interesse, cit, pp. 393-416; inoltre: V. De Giorgi, Vivere per raccontarla: i gruppi intermedi, «Rivista di diritto civile», vol. 58, n 6, 2012, pp. 791-818 e R. Rajan, Il terzo pilastro. La comunità dimenticata da Stato e mercati, Milano, Bocconi Editore, 2019.

55. Per tutti, E. Berti, Il bene di chi? Bene pubblico e bene privato nella storia, a cura di G. Maddalena, Marietti, Genova, 2014, pp. 23-45.

56. L. Ornaghi, Scienza politica e Storia costituzionale. Osservazioni intorno a un sodalizio utile (e possibile), «Giornale di storia costituzionale», vol. 1, n 1, 2001, p. 15.

57. In generale, per alcuni spunti, mi permetto di rinviare a G. Quaglia e M. Rosboch, La forza della società. Comunità intermedie e organizzazione politica, Torino, Aragno, 2018; di estremo rilievo è in proposito la sentenza n. 131/2020 della Corte Costituzionale (relatore Luca Antonini), che osserva come: «Fin da tempi molto risalenti, del resto, le relazioni di solidarietà sono state all'origine di una fitta rete di libera e autonoma mutualità che, ricollegandosi a diverse anime culturali della nostra tradizione, ha inciso profondamente sullo sviluppo sociale, culturale ed economico del nostro Paese. Prima ancora che venissero alla luce i sistemi pubblici di welfare, la creatività dei singoli si è espressa in una molteplicità di forme associative (società di mutuo soccorso, opere caritatevoli, monti di pietà, ecc.) che hanno quindi saputo garantire assistenza, solidarietà e istruzione a chi, nei momenti più difficili della nostra storia, rimaneva escluso. Nella suddetta disposizione costituzionale, valorizzando l'originaria socialità dell'uomo (sentenza n. 75 del 1992), si è quindi voluto superare l'idea per cui solo l'azione del sistema pubblico è intrinsecamente idonea allo svolgimento di attività di interesse generale e si è riconosciuto che tali attività ben possono, invece, essere perseguite anche da una "autonoma iniziativa dei cittadini" che, in linea di continuità con quelle espressioni della società solidale, risulta ancora oggi fortemente radicata nel tessuto comunitario del nostro Paese».

58. Importanti osservazioni in G. Cotta (a cura di), Virtù umane virtù politiche, Milano / Udine, Mimesis, 2020.

\section{RIASSUNTI}

Il breve saggio intende ricostruire in prospettiva storica le vicende dei rapporti fra centro, periferia e comunità negli Stati sabaudi (incluse le aree alpine occidentali), con particolare riguardo al passaggio fra l'Antico regime e il secolo XIX. Le vicende storiche che portano all'Unità sanciscono l'importanza delle scelte dei governi subalpini per lo Stato unitario, fino ai suoi sviluppi contemporanei. Fra questi, meritano una particolare attenzione il tema degli assetti delle istituzioni locali e quello della dimensione comunitaria.

Ce court essai se propose de reconstruire dans une perspective historique l'évolution des relations entre centre, périphérie et communauté dans les États de Savoie (y compris les zones alpines occidentales), avec une attention particulière à la transition entre l'Ancien Régime et le $\mathrm{XIX}^{\mathrm{e}}$ siècle. Les événements historiques qui ont conduit à l'unification de l'Italie confirment 
l'importance des choix effectués par les gouvernements subalpins pour l'État unifié, jusqu'à ses développements contemporains. Parmi ceux-ci, le système des institutions locales et la dimension communautaire méritent une attention particulière.

This short essay proposes to reconstruct in a historical perspective the evolution of the relations between center, periphery and community in the States of Savoy (including the western Alpine areas), with particular attention to the transition between the Ancien Régime and the 19th century. The historical events that led to the unification of Italy confirm the importance of the choices made by the subalpine governments for the unified state, up to its contemporary developments. Among these, the system of local institutions and the community dimension deserve particular attention.

\section{INDICE}

Parole chiave : frontiera, autonomia, comunità

Keywords : border, autonomy, community

Mots-clés : frontière, autonomie, communauté

\section{AUTORE}

\section{MICHELE ROSBOCH}

Università di Torino

michele.rosboch@unito.it 